\title{
Strategies to prevent preterm birth and cerebral palsy: Compliance with current recommendations
}

\author{
Amanda Meyer ${ }^{1}$, Nancy Cipparrone ${ }^{2}$, Andrea Buras ${ }^{1}$, Barbara V. Parilla ${ }^{1^{*}}$ \\ ${ }^{1}$ Department of Obstetrics and Gynecology, Advocate Lutheran General Hospital, Park Ridge, Illinois, USA \\ ${ }^{2}$ Russell Institute for Research \& Innovation, Advocate Lutheran General Hospital, Park Ridge, Illinois, USA \\ Email: "barbara.parilla@advocatehealth.com
}

Received 27 December 2013; revised 18 January 2014; accepted 25 January 2014

Copyright (c) 2014 Amanda Meyer et al. This is an open access article distributed under the Creative Commons Attribution License, which permits unrestricted use, distribution, and reproduction in any medium, provided the original work is properly cited. In accordance of the Creative Commons Attribution License all Copyrights (C) 2014 are reserved for SCIRP and the owner of the intellectual property Amanda Meyer et al. All Copyright (C) 2014 are guarded by law and by SCIRP as a guardian.

\section{ABSTRACT}

Objective: Magnesium sulfate $\left(\mathrm{MgSO}_{4}\right)$ administration to patients with preterm delivery has been associated with a decrease in cerebral palsy. At our institution, a protocol was established regarding the administration of magnesium sulfate for patients in preterm labor at less than 32 weeks of gestation. Despite this protocol, not all eligible patients received this therapy. The purpose of this study was to investigate potential barriers to $\mathrm{MgSO}_{4}$ administration. Methods: A retrospective chart review was performed of those patients who received the diagnosis of "Early Onset Delivery" or "preterm labor" from January through December of 2010, to see what therapies were offered and received. Results: 119 patients met initial criteria. Of those, 68 patients had preterm labor less than 32 weeks. 15 of the 68 patients (22\%) received $\mathrm{MgSO}_{4}$. Of those patients that did not receive $\mathrm{MgSO}_{4}$, only 6 delivered $<32$ weeks. Five were considered eligible. One patient had a relative contraindication to therapy. There were no demographic differences between those patients that received $\mathrm{MgSO}_{4}$, and those that were eligible and did not. Potential barriers included short time frame from presentation to delivery, treatment not considered by healthcare provider, and unanticipated delivery. No patient declined therapy. Conclusions: At our institution, the rate of $\mathrm{MgSO}_{4}$ administration for neuroprotection to eligible candidates was $75 \%$. The subgroup of patients where $\mathrm{MgSO}_{4}$ was not administered in eligible candidates was unanticipated delivery (4), and premature rupture of membranes (1). A 4 gram load of $\mathrm{MgSO}_{4}$ should be attempted prior to delivery of eligible patients, as this strategy has also been shown to be of benefit.

${ }^{*}$ Corresponding author.

\section{KEYWORDS}

Magnesium Sulfate; Neuroprotection; Preterm Delivery

\section{INTRODUCTION}

Preterm delivery (PTD) is the leading cause of neonatal mortality and a significant cause of morbidity including cerebral palsy. Cerebral palsy is strongly linked to prematurity, with infants born at an earlier gestational age at higher risk. Numerous large clinical studies have evaluated the evidence regarding magnesium sulfate, neuroprotection, and preterm births. The ACOG Committee on Obstetric Practice and the Society for Maternal-Fetal Medicine recognize that none of the individual studies found a benefit with regard to their primary outcome. However, the available evidence suggests that magnesium sulfate given before anticipated early preterm birth reduces the risk of cerebral palsy in surviving infants. [1-5]. Our institution elected to use magnesium sulfate for fetal neuroprotection, using the Rouse et al.'s protocol [1].

We sought to evaluate the compliance at our institution of the administration of $\mathrm{MgSO}_{4}$ for neuroprotection in those pregnancies at risk for preterm delivery less than 32 weeks of gestation, as well as to identify barriers potentially contributing to any non-compliance.

\section{METHODS}

This study was a retrospective chart review of those patients who delivered prematurely at less than 32 weeks' gestation at a suburban tertiary care center between January 1, 2010 and December 31, 2010. Study patients were ascertained using the hospital ICD9codes for "preterm labor" and "early onset delivery" ( $<37$ weeks' ges- 
tation). Data abstracted included age, gravity, parity, gestational age at presentation and delivery, whether BMZ, magnesium sulfate or tocolysis was administered, mode of delivery, and if any complications were recorded in the delivery note. If magnesium sulfate was thought to be indicated (preterm labor $\leq 32$ weeks) and not given, an explanation was sought. Preterm labor at our institution requires regular contractions with cervical change, or regular contractions with an already completely effaced or dilated cervix. Our protocol for the administration of magnesium sulfate for neuroprotection was based on the Rouse study [1]. Magnesium sulfate was given as a 6 gram IV load, followed by 2 grams per hour for up to 12 hours. It was discontinued if delivery was not imminent, with retreatment if delivery again appeared imminent at less than 32 weeks. Our first-line tocolytic agent is indomethacin. Therefore, the administration of magnesium sulfate in this population was for neuroprotection only. None of the patients were being treated for pre eclampsia. This study was approved by the Advocate Lutheran General Hospital Institutional Review Board.

\section{STATISTICAL ANALYSES}

Descriptive statistics were reported on categorical data as number and (percent) and continuous data as mean \pm SD. Independent t-test, Chi-square or Fishers Exact test when necessary was performed to measure statistical differences between $\mathrm{MgSO}_{4}$ status (given verses not given). A two-tailed p level of 0.05 was considered statistically significant in all analyses. Analyses were performed with SPSS software (release 19.0, International Business Machines, Chicago).

\section{RESULTS}

Figure 1 is a flow-diagram of patient selection. Of 241 charts reviewed, 119 charts met initial inclusion criteria of preterm labor or preterm delivery. Of these, 51 were excluded for PTD greater than 32 weeks. Twenty were between 32 and 34 weeks, 15 were between 34 and 36 weeks, and 16 had deliveries after 36 weeks. Of the 68 patients with PTL less than 32 weeks, 15 were given $\mathrm{MgSO}_{4}$ and 53 were not. The demographic data is displayed in Table 1. There were no significant demographic differences between those patients that received $\mathrm{MgSO}_{4}$, and those that did not. There was also no significant difference between groups with betamethasone administration and tocolysis, which is reported in Table 2. Of the 53 patients not given $\mathrm{MgSO}_{4}$, only 6 went on to deliver prior to 32 weeks. Of those 6 patients, 1 had a relative contraindication to $\mathrm{MgSO}_{4}$ (severe chronic kidney disease), 3 presented in active labor and were delivered within several hours of admission (mean of 3.5 hours, range 1.2 - 8.8 hours). One antepartum patient
Table 1. Demographic information.

\begin{tabular}{cccc}
\hline $\begin{array}{c}\text { Demographic } \\
\text { data }\end{array}$ & $\begin{array}{c}\text { Magnesium } \\
\text { sulfate } \\
\text { given } \\
(\mathrm{N}=12)\end{array}$ & $\begin{array}{c}\text { Magnesium } \\
\text { sulfate } \\
\text { not given } \\
(\mathrm{N}=51)\end{array}$ & P value \\
\hline Maternal age & $30.29+/-7.09$ & $30.04+/-5.9$ & 0.895 \\
Race & $7(58.3)^{\dagger}$ & $35(68.6)$ & 0.513 \\
caucasian & $5(41.7)$ & $16(31.4)$ & \\
other $^{\ddagger}$ & & & \\
Insurance & $7(58.3)$ & $36(70.6)$ & 0.496 \\
private & $5(41.7)$ & $15(29.4)$ & \\
public aid & &
\end{tabular}

${ }^{*}$ Mean $\pm \mathrm{SD} ;{ }^{\dagger} \mathrm{N}(\%) ;{ }^{\ddagger}$ Includes the following races: Hispanic, African American, Pacific Islander, Indian, Asian (not Indian), and Unknown.

Table 2. Pregnancy and delivery data.

\begin{tabular}{cccc}
\hline $\begin{array}{c}\text { Pregnancy } \\
\text { outcome }\end{array}$ & $\begin{array}{c}\text { Magnesium } \\
\text { sulfate } \\
\text { given } \\
(\mathrm{N}=15)\end{array}$ & $\begin{array}{c}\text { Magnesium } \\
\text { sulfate } \\
\text { not given } \\
(\mathrm{N}=53)\end{array}$ & P value \\
\hline $\begin{array}{c}\text { Gestational age } \\
\text { at delivery }\end{array}$ & $37.02+/-1.67$ & $35.69+/-3.23$ & 0.329 \\
$\begin{array}{c}\text { Betamethasone } \\
\text { given }\end{array}$ & $14(100)^{\dagger}$ & $50(94.3)$ & $>0.99$ \\
Yes & $0(0)$ & $3(5.7)$ & \\
No & & & \\
Tocolysis given & $15(100)$ & $46(86.8)$ & 0.334 \\
Yes & $0(0)$ & $7(13.2)$ & \\
No & & & \\
\hline
\end{tabular}

${ }^{*}$ Mean $\pm \mathrm{SD}{ }^{\dagger} \mathrm{N}(\%)$.

with twins and preterm labor considered stable went on to have PPROM and was delivered by cesarean for malpresentation approximately 3 hours thereafter. Another antepartum patient previously stable went into active labor and delivered by spontaneous vaginal delivery within 20 minutes of assessment. No patient declined $\mathrm{MgSO}_{4}$ therapy.

\section{DISCUSSION}

At our institution, the rate of $\mathrm{MgSO}_{4}$ administration for neuroprotection to eligible candidates was 75\%. Unanticipated delivery was noted primarily in patients with progressive preterm labor where a 6 gram load followed by 2 grams per hour was not given either because the physician did not consider the therapy, or did not think there was ample time to administer the full therapy based on our protocol. Under those circumstances, a 4 gram load of $\mathrm{MgSO}_{4}$ should be attempted prior to delivery of eligible patients, as this strategy has also been shown to be of benefit [5]. The time from presentation to delivery is often the most adequate for this intervention. Extra effort towards aggressively administering $\mathrm{MgSO}_{4}$ boluses to inpatients with a change in status should be emphasized. 


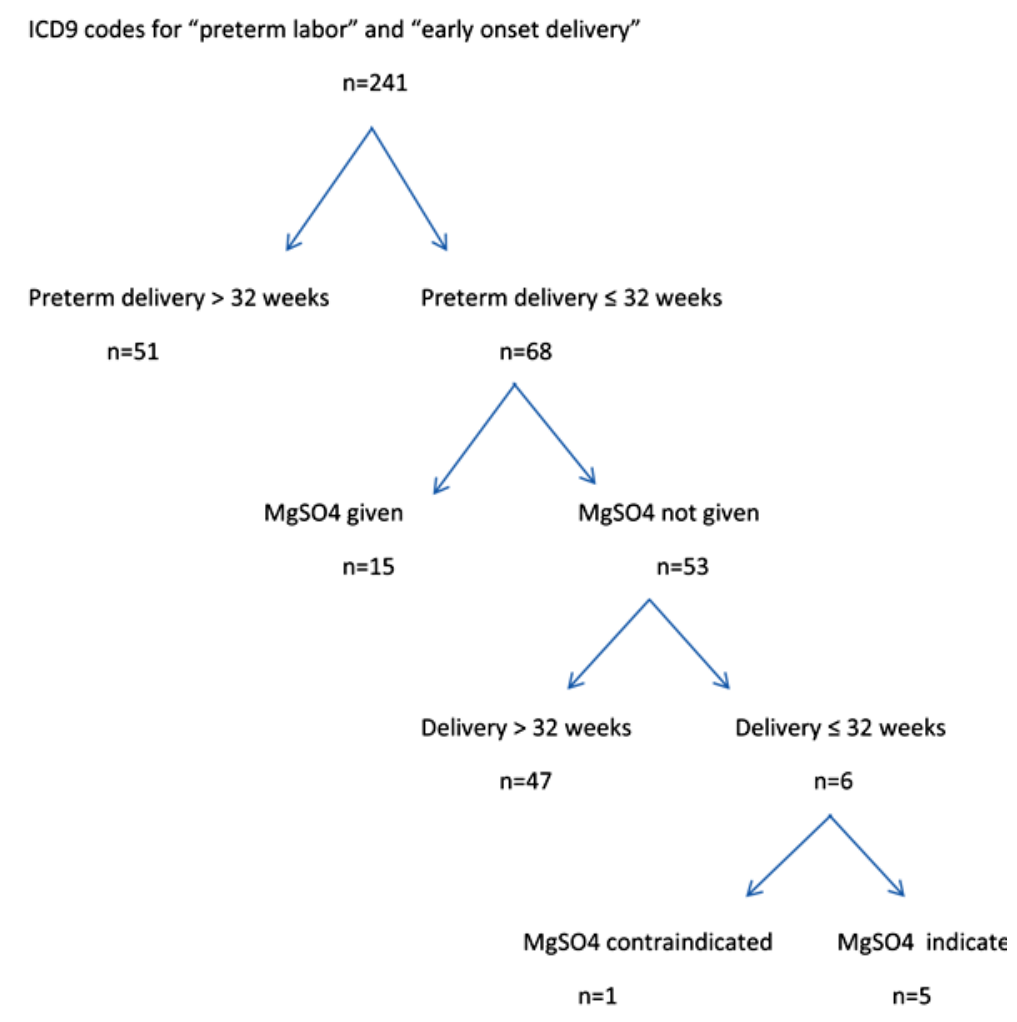

Figure 1. Patient selection.

The prior studies reporting on the use of magnesium sulfate for neuroprotection all used different regimens. Therefore, it is not clear which regimen if any is superior. Consequently, it is not evident whether any exposure verses a therapeutic magnesium level in the neonate at the time of delivery confers the protection. It is unlikely that further studies addressing the question of timing and dosing will be performed. Therefore, a reanalysis of previous data to try to address this question would be very useful. We chose the Rouse protocol based on the most significant RR of 0.55 for cerebral palsy. This approach was later endorsed in a clinical opinion regarding magnesium sulfate for fetal neuroprotection [6]. The authors outlined an approach that not only identified the specific patients who qualify for magnesium sulfate therapy, but also provided a treatment algorithm which addressed retreatment and concomitant tocolysis. Interestingly, Rouse and colleagues presented an abstract on $\mathrm{MgSO}_{4}$ dose and timing, umbilical cord $\mathrm{Mg}^{++}$concentration and the relationship to cerebral palsy [7]. The concentration of $\mathrm{MgSO}_{4}$ was negatively related to $\mathrm{CP}$, but it was not statistically significant likely due to low numbers. They concluded that their analyses did not support any specific modification of the intravenous $\mathrm{MgSO}_{4}$ dosing regimen that reduced the rate of cerebral palsy in the original clinical trial.

Lastly, this important therapy should not be withheld secondary to the recent US Food and Drug Administra- tion's warning against the prolonged use of magnesium sulfate [8]. Based on this warning, the drug classification was changed from Category A to Category D and the labeling was changed to include this new warning information. However, the US Food and Drug Administration's change in classification addresses an unindicated and nonstandard use of magnesium sulfate in obstetric care, specifically, magnesium sulfate for more than 5 - 7 days to stop preterm labor. The American College of Obstetricians and Gynecologists and the Society for Maternal-Fetal Medicine continue to support the short-term use, usually less than 48 hours, of magnesium sulfate in obstetric care for appropriate conditions and for appropriate durations of treatment which includes the prevention of seizures in women with preeclampsia or eclampsia, fetal neuroprotection before anticipated preterm delivery less than 32 weeks of gestation, and short-term prolongation of pregnancy to allow for the administration of antenatal corticosteroids in pregnant women between 24 and 34 weeks of gestation who are at risk of preterm delivery within 7 days.

\section{REFERENCES}

[1] Rouse, D.J., Hirtz, D.G., Thom, E., et al. (2008) A randomized, controlled trial of magnesium sulfate for the prevention of cerebral palsy. The New England Journal of Medicine, 359, 895. 
http://dx.doi.org/10.1056/NEJMoa0801187

[2] Crowther, C.A., Hille, J.E., Doyle, L.W. and Haslam, R.R. (2003) Effect of magnesium sulfate given for neuroprotection before preterm birth: A randomized controlled trial. JAMA, 290, 2669-2676. http://dx.doi.org/10.1001/jama.290.20.2669

[3] Marret, S., Marpeau, L., Follet-Bouhamed, C., et al. (2008) Effect of magnesium sulphate on morality and neurologic morbidity of the very-preterm newborn (of less than 33 weeks) with two-year neurological outcome: Results of the prospective PREMAG trial. Gynécologie Obstétrique \& Fertilité, 36, 278-288. http://dx.doi.org/10.1016/j.gyobfe.2008.01.012

[4] Costantine, M.M. and Weiner, S.J. (2009) Eunice Kennedy Shriver National Institute of Child Health and Human Development Maternal-Fetal Medicine Units Network. Effects of antenatal exposure to magnesium sulfate of neuroprotection and mortality in preterm infants: A meta-analysis. Obstetrics \& Gynecology, 114, 354. http://dx.doi.org/10.1097/AOG.0b013e3181ae98c2

[5] American College of Obstetricians and Gynecologists
(2010) Magnesium sulfate before anticipated preterm birth for neuroprotection. Committee Opinion No. 455. Obstetrics \& Gynecology, 115, 669-671. http://dx.doi.org/10.1097/AOG.0b013e3181d4ffa5

[6] Reeves, S.A., Gibbs, R.S. and Clark, S.L. (2011) Magnesium for fetal neuroprotection. American Journal of Obstetrics \& Gynecology, 204, e1-4.

[7] Rouse, D.J. (2008) Magnesium sulfate $\left(\mathrm{MgSO}_{4}\right)$ dose and timing, and umbilical cord $\mathrm{Mg}++$ concentration: Relationship to cerebral palsy (CP). American Journal of $\mathrm{Ob}$ stetrics \& Gynecology, 199.

[8] Food and Drug Administration (2013) FDA recommends against prolonged use of magnesium sulfate to stop preterm labor due to bone changes in exposed babies. FDA Drug Safety Communication. Silver Springs, FDA. http://www.fda.gov/downloads/Drugs/DrugSafety/UCM3 53335.pdf 